\title{
Performance Investigation of Ground Cooling for the Airbus A380 in the United Arab Emirates
}

\section{Mr Brendan Savio Aranjo}

Mechanical Engineering, School of Engineering and Physical Sciences, Heriot-Watt University, Dubai International Academic City, Dubai, United Arab Emirates

\section{Dr Ben Richard Hughes}

Dubai Energy Research Group, School of Engineering and Physical Sciences, Heriot-Watt University, Dubai International Academic City, Dubai, United Arab Emirates

\section{Mr Hassam Nasarullah Chaudry}

Dubai Energy Research Group, School of Engineering and Physical Sciences, Heriot-Watt University, Dubai International Academic City, Dubai, United Arab Emirates

\begin{abstract}
A combination of the United Arab Emirates (UAE) climate and the increased size of the super jumbo, Airbus A380, have exceeded the working capacity for current ground cooling techniques. These are evident when the aircraft is being prepared for flight and when in the hanger under maintenance with internal cabin temperatures reported at above $30^{\circ} \mathrm{C}$. The existing system used, delivers air at $11.6^{\circ} \mathrm{C}$ after which the cabin temperature is still at a high temperature of $31^{\circ} \mathrm{C}$ and unable to cool down due to the temperature rises caused by climate conditions and heat dissipation from the electronics in the cabin. The CFD based temperature profile results highlighted that a decrease in inlet temperature to $-18^{\circ} \mathrm{C}$ at a constant pressure and mass flow rate is sufficient to provide efficient cooling to the cabin at $22^{\circ} \mathrm{C}$. Boundary conditions are determined to specify a new effective cooling system and resolve the ground cooling issue.
\end{abstract}

Keywords: Computational Fluid Dynamics, Analysis, Energy, Cooling, Pre-Conditioned Air, Thermal Comfort 


\section{INTRODUCTION}

Emirates was the first airline to place an order and is currently the largest operator of the Airbus A380, with 15 in service out of its total of 90 on order, which is the largest amount of any carrier. Emirates uses the original A380-800 configuration that carries 517 passengers in a threeclass configuration for long range flights or 600 passengers in a two-class configuration for medium range flights [1] as shown in Table 1.

Table 1: Airbus A380 Aircraft Specifications [2] [3]

\begin{tabular}{|l|ll|}
\hline Length & $238 \mathrm{ft} 1$ in & $72.8 \mathrm{~m}$ \\
Wingspan & $261 \mathrm{ft} 8 \mathrm{in}$ & $79.8 \mathrm{~m}$ \\
Height & $79 \mathrm{ft} 7$ in & $24.1 \mathrm{~m}$ \\
Cabin Length & $172 \mathrm{ft} 5 \mathrm{in}$ & $52.55 \mathrm{~m}$ \\
Cabin Width & $21 \mathrm{ft} 7$ in & $6.58 \mathrm{~m}$ \\
Cabin Height & 5930 sq. ft. & $551 \mathrm{sq} . \mathrm{m}$. \\
& 3 class -489 Ultra Long Range \& 517 \\
Passenger Capacity & Long Range \\
& 2 class -600 Medium Range \\
Certified Ceiling & $43000 \mathrm{ft}$ & $13100 \mathrm{~m}$ \\
Take Off Distance & $9450 \mathrm{ft}$ & $2880 \mathrm{~m}$ \\
Landing Distance & $6660 \mathrm{ft}$ & $2030 \mathrm{~m}$ \\
Maximum Take Off Weight & $1,235,000 \mathrm{lbs}$ & $560000 \mathrm{~kg}$ \\
Maximum Landing Weight & $851000 \mathrm{lbs}$ & $386000 \mathrm{~kg}$ \\
\hline
\end{tabular}

Emirates airlines operate out of Dubai where the temperature at the airport during the summer months is at an estimated average of $37^{\circ} \mathrm{C}$, with temperatures sometimes exceeding $40^{\circ} \mathrm{C}$.

The combination of the climatic conditions and the size of the A380 have created cooling issues with the aircraft when on ramp at concourse being prepared for flight and when in the hanger under maintenance, using current Pre-Conditioned Air (PCA) systems. When the aircraft is in the air, the external airflow cools the skin of the aircraft and the Auxillary Power Unit (APU) cools the cabin. But when the aircraft is stationary on the ground and the APU switched off, external cooling needs to be provided.

The high external temperature heats up the skin of the aircraft during pre-flight procedures. Emirates Engineering provides cooling with external PCA supply units which goes through long lengths of flexible ducting before reaching the cabin. Because of the sheer size of the aircraft, this cooling method is inadequate to cool the aircraft even with an additional PCA unit attached. Therefore the APU is switched on for effective cooling, but this is very inefficient in terms of fuel usage. The APU uses between 100-600 litres of fuel per hour to cool the aircraft cabin, and because the alternative refrigeration systems cannot cool the large aircraft due to the external temperatures, the APU is constantly deployed. Considering the number of Airbus A380s at the Dubai airport and Emirates maintenance hangar, a considerable amount of fuel is consumed per hour to run the APU which is unsustainable in the current aviation market [4]. 
A similar problem is faced while the aircraft is under maintenance in the hangar. The existing PCA Unit is unable to cool the A380 effectively. Cooling from the PCA unit is supplied using two supply ducts, and for cooling the A380 an additional PCA unit with two extra supply ducts is used. During maintenance, pre-conditioned air from the units is supplied directly into the cabin through the doors. Substantial cooling is lost from the ducting due to the PCA unit being more than 75 meters away from the aircraft.

Emirates Engineering uses ADX type PCA units from CIAT which are self-contained, aircraft cabin air-designed to provide the airflow and pressure required for cooling or heating and ventilation of parked commercial passenger aircraft. The PCA units are designed as standard for installation outdoors at point of use (POU) without any special weather protection [5] shown in Figure 2.
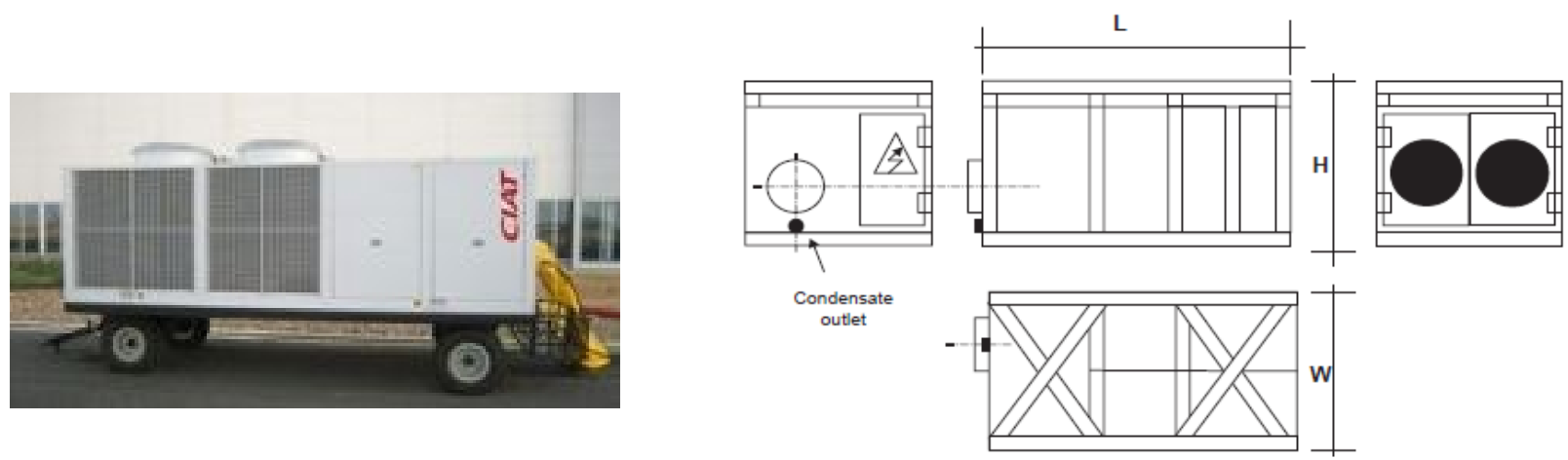

Figure 2: CIAT ADX type, Pre Conditioned Air Unit. [5]

High external climate conditions have been shown to affect the temperature of aircraft in the hangar, leading to problems during maintenance. The AMECO-A380 aircraft hangar at the Beijing Capital International Airport was under study by Yongzhong et al. [6] as a practical example to calculate the temperature rise taking into consideration, solar radiation and heat convection. When the aircraft hangar door is kept partially open, warm air from outside flows into the hangar but the hot air between the top of the door and the top of the aircraft hangar roof will not circulate well. Therefore the temperature of the upper chords of the roof will rise by $2^{\circ} \mathrm{C}$ and the lower chords by $3.2^{\circ} \mathrm{C}$ with reference to the climate temperature. The results of the study show that the temperature effect will be considerable for long-span hangars built to accommodate the Airbus A380.

The A380 is so large that the amount of cooling given for standard aircraft is not sufficient. The study investigated this area to identify the cooling inefficiencies by creating simulation models of the structure of the aircraft and map the temperatures with respect to external conditions using the 3D Computer Aided Design (CAD) software; Solid Edge ST and the CFD software; Ansys 12.1. Current cooling strategies were then evaluated for their effectiveness and accordingly an optimized cooling system was identified and simulated. 
Zhao et al. [7] performed a study on the off-design performance and dynamic response of an aircraft Environmental Control System (ECS). The ECS in the study utilized a bootstrap air cycle refrigeration system with high pressure water separation. Static and dynamic tests were carried out using air streams from a low pressure compressor system and data was collected using sensors. The results acquired from the testing validate that the performance of the ECS can meet the prerequisites of the design. The final results show that the off-design performance and dynamic response should be considered in the design of the ECS to improve its performance and effectiveness.

Perez-Grande and Leo [8] worked on optimizing the ECS of a commercial aircraft. In this work, the cross-flow heat exchangers incorporated into the ECS of the commercial aircraft were optimized with weight and minimum entropy generation simultaneously to find the major geometric characteristics. The ECS was based on Brayton inverse cycle, where two air streams were involved. To obtain the value of the entropy generation rate of the global system and the total value of the heat exchangers, an analytical model was developed, which allowed the calculation of two objective functions for different values of the parameters. It was concluded that trade-off solutions turned out to be the adequate ones for optimization problems where devices under interest were part of a complex system.

Perez-Grande and Leo [9] performed a thermo-economic analysis on the ECS of a commercial aircraft. In this work, an application of thermo-economics to aeronautics was carried out, where based on the second law of thermodynamics, methods have been developed to assign costs and optimize thermal systems from an economic point of view. A cost balance was applied to the ECS as a component, and the unit cost of the conditioning stream entering the aircraft cabin was obtained for a range of aircraft engine bleed pressure values. . From the results obtained it was concluded that the operating costs of the ECS were too high due to the fact that it was necessary to transport the system. The cost of carrying the excess weight of the heat exchangers and the extra fuel necessary to compress bled air was taken in dollars per metric ton and kilometer.

Kuhn et al. [10] performed a study of forced and mixed convection in a geometric model of a passenger aircraft. A geometric model was created representing a cabin section of the upper deck of the Airbus A380. Particle Image Velocimetry (PIV) and temperature field measurements were carried out on a cross sectional plane of the geometric model. A CFD model of the cabin section was developed, and the turbulent velocity streams and temperature contours were analyzed. The study confirmed that the flow field in the aircraft cabin section was affected by the negative buoyancy forces acting on the air jets and interaction of thermal plumes with the supplied air jets. From the CFD analysis results it was established that a personalized air distribution system gives the best performance as a result of the improved air quality. 
Zhang and Chen [11] investigated air distribution systems for commercial aircraft cabins. An under-floor air delivery system and a personalized system was proposed to enhance the air distribution system of the Boeing 767 aircraft cabin that was selected for the study. CFD analysis using the FLUENT code was undertaken to calculate the air velocity and temperature distributions as well as the carbon dioxide concentration levels using the personalized air distribution system and under floor displacement. The CFD results provided the airflow streamlines and the supply of temperature, pressure, carbon dioxide concentration and air velocity. The results concluded that the ideal aircraft cabin environment can be attained by utilizing a personalized air distribution system.

Zhang et al. [12] carried out a study on an under-aisle air distribution system of commercial aircraft cabins. CFD analysis was used to design a new under-aisle air distribution system to improve the air humidity level while concurrently confining air mixing. The new system supplied dry air through the aircraft cabins while humidified air was delivered through both under aisles simultaneously. The performance of the new system was studied using CFD after the system was validated in a workshop with under-floor displacement ventilation. From the results obtained, the new system could enhance the relative humidity by $10 \%-20 \%$ and reduce the carbon dioxide concentrations by $30 \%$ in contrast to the existing system.

Gunther et al. [13] investigated numerical and experimental simulations of idealized aircraft cabin flows. In a generic mock-up, the airflow through the aircraft cabin was investigated using StarCD to perform numerical simulations so as to predict the airflow and optimize the cabin design with regards to thermal comfort. Experimental investigations were also conducted utilizing PIV and thermography. Comparisons between the experimental results and the simulations showed a satisfactory agreement. For the simulations, two turbulence models were used because of their better capacity to depict the jet diffusion and separation.

Park et al. [14] carried out a study on the overall thermal comfort of passengers in an aircraft cabin. A subject study was carried out in a replicated aircraft cabin using forty different test subjects. The percentage of thermal dissatisfied test persons was higher than predicted due to the local thermal discomfort. The statistical analysis specifies that the overall thermal discomfort is a result of the test subject's local thermal perception and overall thermal sensation.

The studies carried out by Kuhn, Zhang et al. use a CFD program to analyse the airflow distribution in the cabin of the Airbus A380 and Boeing 767 respectively. It was demonstrated that several effects have a large impact on the flow field in an aircraft cabin. A thorough examination of these effects is very important for designing the air conditioning of a passenger aircraft. The study on under-aisle air distribution system by Zhang utilizes the same CFD program (FLUENT) that was utilized in this research. Unlike Zhang's study which used a box shaped manikin schematic in the program to study the global airflow in the space, This work created an improved schematic using accurate complex geometry of the aircraft cabin using Solid Edge ST before using FLUENT to analyse the airflow and temperature. 
Additionally, this work calculated the heat dissipation required based on occupancy and equipment usage. The investigation on experimental and numerical simulations of idealized aircraft cabin flows by Gunther et al. demonstrates that CFD analysis allows for optimization of the cabin design and indicates that higher order low Reynolds number turbulence models are the most suitable to predict airflow through the A380 cabin mock-up.

\section{CFD MODELING}

The geometry of the aircraft cabins were designed using Solid Edge ST with the dimensions provided by Emirates airline. Ansys 12.1 is used to mesh the geometry and export it into FLUENT, which is used to analyse the change in temperature through the aircraft cabins under set boundary conditions. Post processing software is then used to simulate the results and generate temperature contours and velocity streamlines through the cabins.

The dimensions for the cabin and the seating layout were accurately recreated from available resources [15]. Figure 3 shows the complete geometry model in 3D view designed using the 3D CAD modeling software, Solid Edge ST for the business class cabin area.
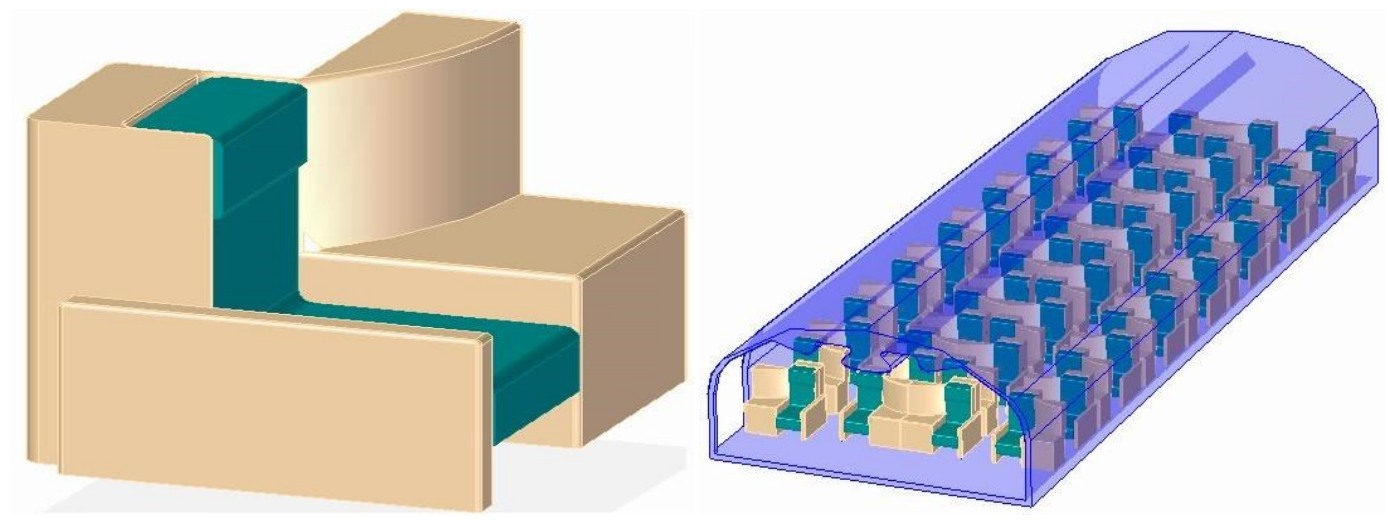

Figure 3. CAD representation of the Airbus A380 business class cabin

Figure 4 shows the complete geometry model in 3D view designed using the 3D CAD modeling software, Solid Edge ST for the economy class cabin. 

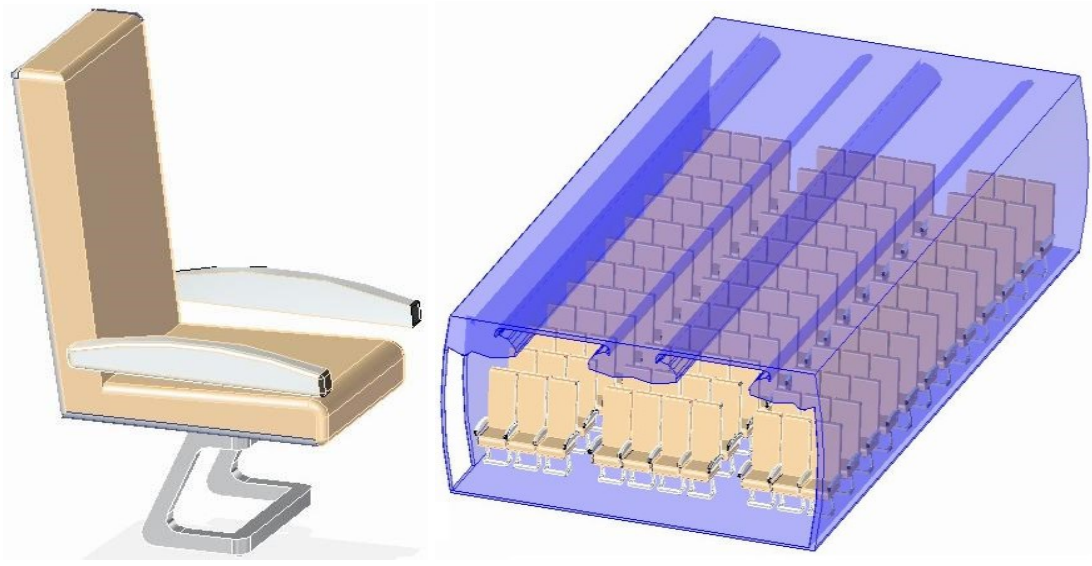

Figure 4. CAD representation of the Airbus A380 economy class cabin

The Solid Edge geometry was simplified and only a section of each cabin was imported into the Ansys 12.1 workbench to limit the number of cells for efficient computation. Within the workbench, the ANSYS Design Modeller was used to create an enclosure around the geometry to initiate a flow from the front face of the enclosure marked as the 'Velocity Inlet.' The opposite face of the enclosure was marked as the 'Velocity Outlet' and the other faces were marked as 'Symmetry'.

\subsection{The business cabin}

A section of the business cabin was modeled with an enclosure around the seats to model the volume of air through the cabin. The enclosure was created with a length of $9.35 \mathrm{~m}$, width $5.92 \mathrm{~m}$ and height $2.60 \mathrm{~m}$, shown in Figure 5.

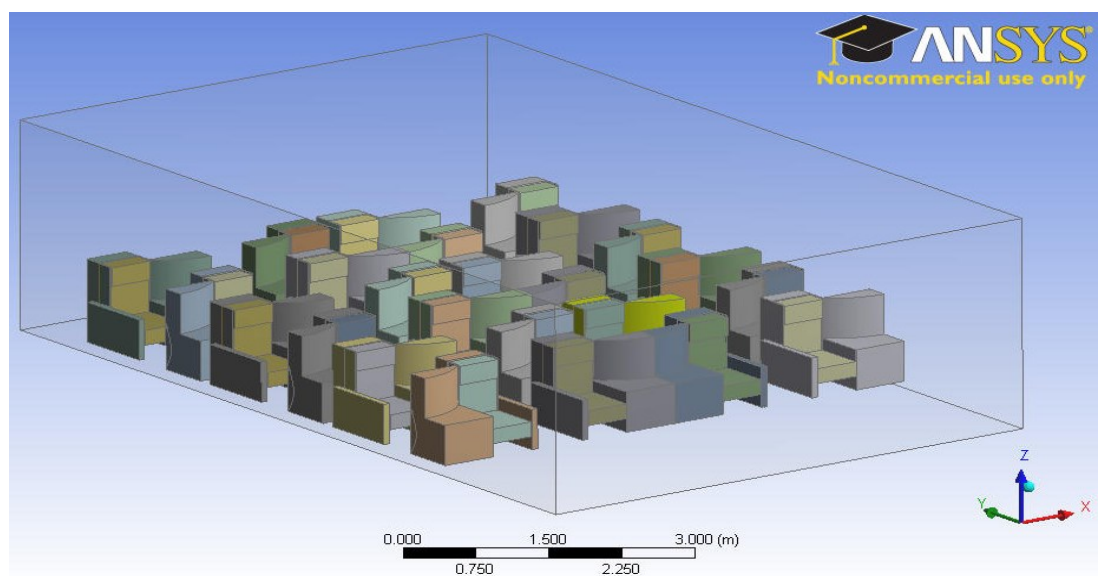

Figure 5. CFD geometrical model of the business cabin 


\subsection{The economy cabin}

A section of the economy cabin was modeled with an enclosure around the seats to model the volume of air through the cabin. The enclosure was created with a length of $7.24 \mathrm{~m}$, width $6.34 \mathrm{~m}$ and height $2.37 \mathrm{~m}$, shown in Figure 6.

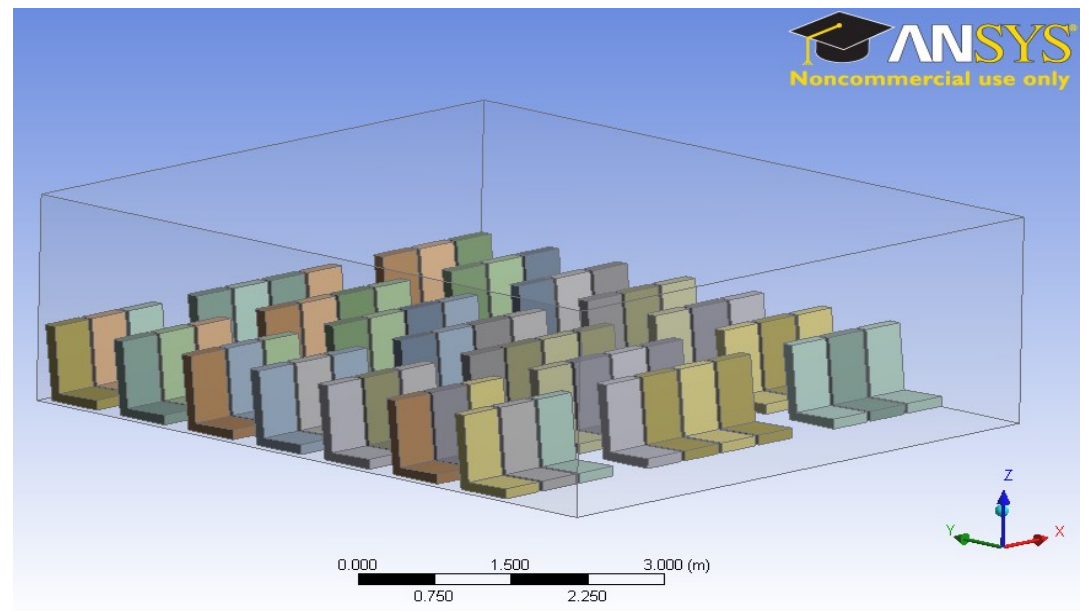

Figure 6. CFD geometrical model of the economy cabin

\subsection{Boundary Conditions}

Boundary conditions specify the flow and thermal variables on the boundaries of the geometrical model. In the ANSYS Design Modeler, simple boundary conditions were applied to the enclosure surrounding the model. The front face of the model was set as the 'velocity inlet' to simulate airflow through the cabin, and the opposite face of the enclosure was set as the 'pressure outlet.' The rest of the faces were to have no effect on the flow so they were set as 'symmetry' to have a single-directed flow from the inlet to the outlet.

The Geometrical models were then imported into FLUENT and boundary conditions were set for the 'Velocity Inlet', 'Pressure outlet', volume of air and the cabin seats. A standard k-epsilon turbulence model was enabled to simulate the airflow through the cabin. The air flow was given a mass flow rate of $6.5 \mathrm{~kg} / \mathrm{s}$ from the velocity inlet, the same mass flow rate distributed from the PCA outlet. Considering the flow expansion over the cross-sectional area of the cabin after being distributed from the PCA outlet, the velocity is reduced to an estimated $0.35 \mathrm{~m} / \mathrm{s}$.

$\dot{m}=\rho v A$, $6.5=1.2 \times \mathrm{v} \times 15.3$, $\mathrm{v}=0.35 \mathrm{~m} / \mathrm{s}$ 
The initial gauge pressure was taken as $8500 \mathrm{~Pa}$, which is the maximum allowed pressure for the given flow rate. The temperature of the volume of air in the cabin was set at $311 \mathrm{~K}$ which is the highest average temperature rise through the cabin due to the effect of summer climate temperatures in Dubai.

When the aircraft sits in the hangar or at concourse before the passengers have boarded, the electronics are sometimes switched on. With the heat gain from the 17 inch business cabin screens at $70 \mathrm{~W}$ and the area of each seat at $1.15 \mathrm{~m}^{2}$, the seats through the business cabin were given an estimated heat flux of $60.36 \mathrm{~W} / \mathrm{m}^{2}$ to simulate the dissipation of heat from the LCD screens and the electronics in the cabin, while the economy cabin seats were given an estimated heat flux of $99.63 \mathrm{~W} / \mathrm{m}^{2}$ with the heat gain from the 10.5 inch economy cabin screens at $55 \mathrm{~W}$ and the area of each seat at $0.552 \mathrm{~m}^{2}$. The summary of boundary conditions is shown in table 2 and table 3.

\section{Table 2. Boundary conditions for} business cabin model

\begin{tabular}{ll}
\hline \multicolumn{1}{c}{ Boundary Conditions } & \multicolumn{1}{c}{ Value } \\
\hline Inlet Mass Flow Rate & $6.5 \mathrm{Kg} / \mathrm{s}$ \\
Initial Gauge Pressure & $8500 \mathrm{~Pa}$ \\
Outlet Pressure & $0 \mathrm{~Pa}$ \\
Model & $\begin{array}{l}\mathrm{K}-E p s i l o n ~-~ \\
\text { Standard }\end{array}$ \\
Inlet Temperature & $286.4 \mathrm{~K}$ \\
Free Stream Temperature & $311 \mathrm{~K}$ \\
Fluid & Air \\
Wall Heat Flux & $60.36 \mathrm{~W} / \mathrm{m}^{2}$ \\
Wall Heat Generation Rate & $60.36 \mathrm{~W} / \mathrm{m}^{3}$ \\
\hline
\end{tabular}

Table 3. Boundary conditions for economy cabin model

\begin{tabular}{ll}
\hline \multicolumn{1}{c}{ Boundary Conditions } & \multicolumn{1}{c}{ Value } \\
\hline Inlet Mass Flow Rate & $6.5 \mathrm{Kg} / \mathrm{s}$ \\
Initial Gauge Pressure & $8500 \mathrm{~Pa}$ \\
Outlet Pressure & $0 \mathrm{~Pa}$ \\
Model & $\begin{array}{l}\mathrm{K}-E p s i l o n- \\
\text { Standard }\end{array}$ \\
Inlet Temperature & $260 \mathrm{~K}$ \\
Free Stream Temperature & $311 \mathrm{~K}$ \\
Fluid & Air \\
Wall Heat Flux & $99.63 \mathrm{~W} / \mathrm{m}^{2}$ \\
Wall Heat Generation Rate & $141.75 \mathrm{~W} / \mathrm{m}^{3}$ \\
\hline
\end{tabular}




\subsection{Grid Generation}

The mesh arrangement for the business cabin model consisted of 55084 nodes and 148582 Tetrahedra mesh elements, and the mesh arrangement for the economy cabin model consisted of 50507 nodes and 181431 Tetrahedra mesh elements. The economy cabin overall has a higher number of elements due to having more seats per row shown in Figures 7 and 8.

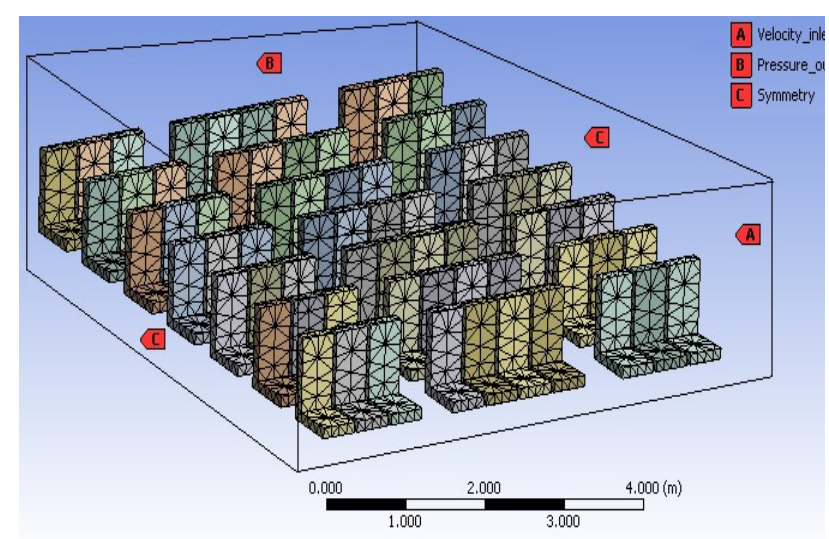

\begin{tabular}{c|ccc}
\hline Domain & Nodes & Elements & Tetrahedra \\
\hline $\begin{array}{c}\text { All } \\
\text { Domains }\end{array}$ & 55084 & 157734 & 148582 \\
\hline
\end{tabular}

Figure 7 Economy cabin CFD mesh model

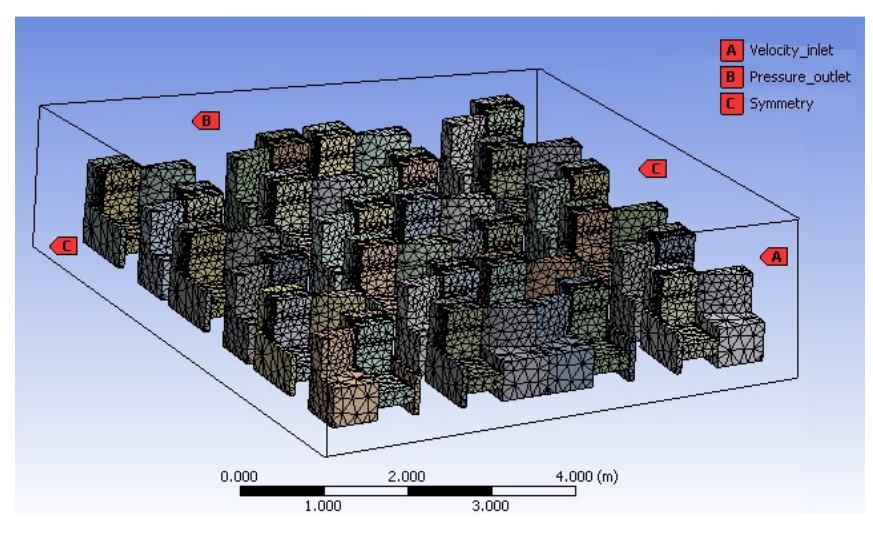

\begin{tabular}{c|ccc}
\hline Domain & Nodes & Elements & Tetrahedra \\
\hline $\begin{array}{c}\text { All } \\
\text { Domains }\end{array}$ & 50507 & 181431 & 181431 \\
\hline
\end{tabular}

Figure 8. Business cabin CFD mesh model

Grid verification is a process of removing computational uncertainties through iterative grid solutions. The hp-method grid adaption technique used requires the use of different mesh sizes and higher order approximations [16]. This technique uses a post-processing error indicator as opposed to other methods which rely on a priori error indicator.

\subsubsection{Economy cabin model}

The initial grid was coarse and served as a benchmark for the successive re-meshing of the model to identify the reduction or increase in post-processing error indicator, which in this investigation was the average internal temperature, shown in Table 4 and summarized in Figure 9. 
Table 4. Error estimate table of the economy cabin model

\begin{tabular}{lccccc}
\hline Nodes & Elements & $\begin{array}{c}\text { Relevance } \\
\text { center }\end{array}$ & Smoothing & $\begin{array}{c}\text { Average } \\
\text { Temperature (K) }\end{array}$ & $\begin{array}{c}\text { Percentage } \\
\text { Error }\end{array}$ \\
\hline $\mathbf{3 1 0 5 6 9}$ & 177082 & Coarse & High & 291.4 & - \\
$\mathbf{3 1 3 6 3 8}$ & 179823 & Coarse & Low & 291.4 & $0 \%$ \\
$\mathbf{3 1 6 4 3 2}$ & 181431 & Coarse & Medium & 295.9 & $4.97 \%$ \\
$\mathbf{4 0 6 1 0 5}$ & 229842 & Fine & High & 293.3 & $9 \%$ \\
$\mathbf{4 0 9 5 0 6}$ & 232613 & Fine & Medium & 289.6 & $13 \%$ \\
$\mathbf{4 5 3 2 4 1}$ & 253578 & Fine & Low & 291.4 & $6 \%$ \\
$\mathbf{4 8 1 8 9 0}$ & 270727 & Medium & High & 291.8 & $1 \%$ \\
$\mathbf{4 8 6 6 7 8}$ & 274396 & Medium & Medium & 291.6 & $0 \%$ \\
\hline $\mathbf{4 9 3 9 5 8}$ & 280175 & Medium & Low & 291.4 & $1 \%$ \\
\hline
\end{tabular}

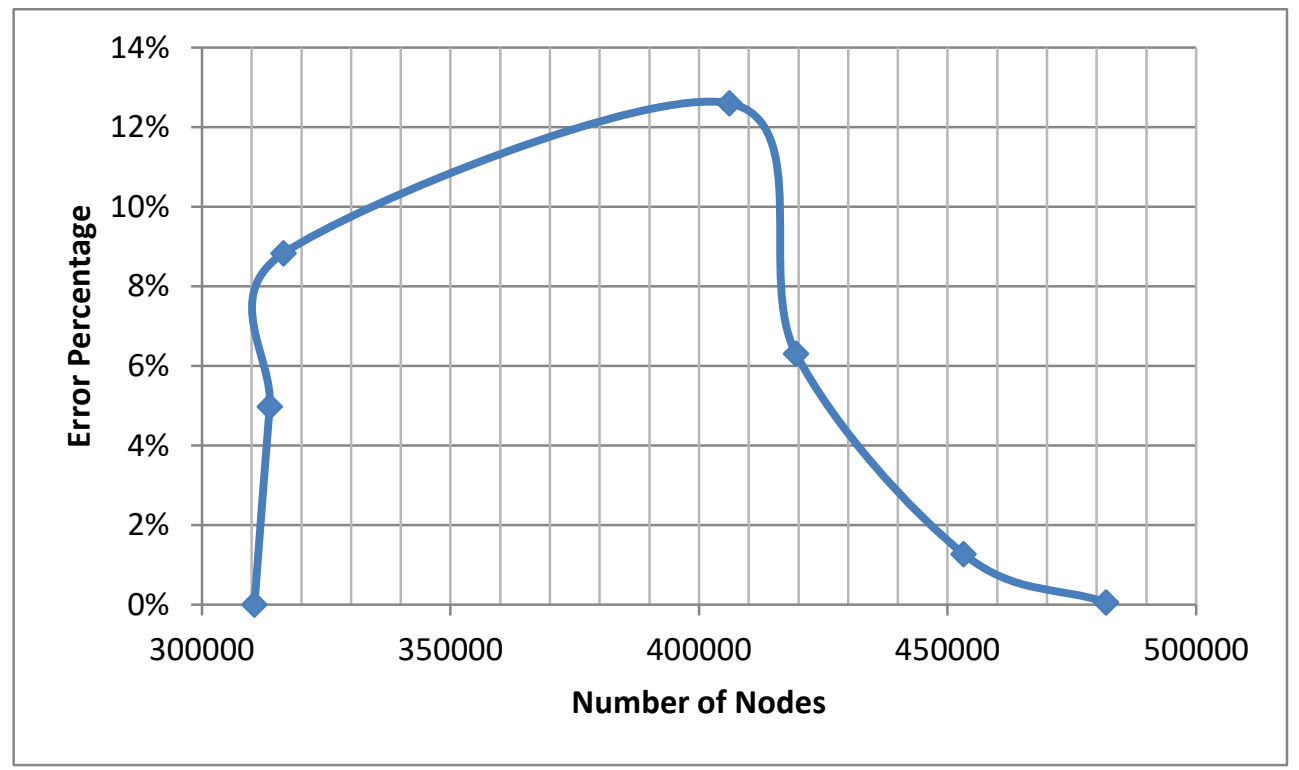

Figure 9. Error reductions through successive hp-method grid adaption techniques (Economy Cabin model)

From the grid adaption the number of nodes was selected as 486,678 with 274,396 elements, as this provided a balance of computational accuracy and efficiency.

\subsubsection{Business cabin model}

The initial grid was coarse and served as a benchmark for the successive re-meshing of the model to identify the reduction or increase in post-processing error indicator, which in this investigation was the average internal temperature, shown in Table 5 and summarized in Figure 10 . 
Table 5. Error estimate table of the business cabin model

\begin{tabular}{lccccc}
\hline Nodes & Elements & Relevance center & Smoothing & $\begin{array}{c}\text { Average } \\
\text { Temperature } \\
\text { (K) }\end{array}$ & $\begin{array}{c}\text { Percentage } \\
\text { error }\end{array}$ \\
\hline $\mathbf{1 5 0 5 7 9}$ & 91301 & Coarse & High & 282.3 & - \\
$\mathbf{1 5 5 6 4 8}$ & 97208 & Coarse & Low & 282.3 & $0 \%$ \\
$\mathbf{1 5 9 0 4 1}$ & 99935 & Coarse & Medium & 284.5 & $8 \%$ \\
$\mathbf{2 4 8 2 5 1}$ & 144061 & Fine & High & 283.3 & $4 \%$ \\
$\mathbf{2 5 7 6 3 9}$ & 150974 & Fine & Low & 282.3 & $3.50 \%$ \\
$\mathbf{2 5 7 8 7 1}$ & 151117 & Fine & Medium & 282.1 & $1 \%$ \\
$\mathbf{3 0 0 8 7 3}$ & 150935 & Medium & High & 282.3 & $1 \%$ \\
$\mathbf{3 0 9 9 8 5}$ & 157734 & Medium & Medium & 282.3 & $0 \%$ \\
$\mathbf{3 1 7 2 6 5}$ & 163513 & Medium & Low & 282.3 & $0 \%$ \\
\hline
\end{tabular}

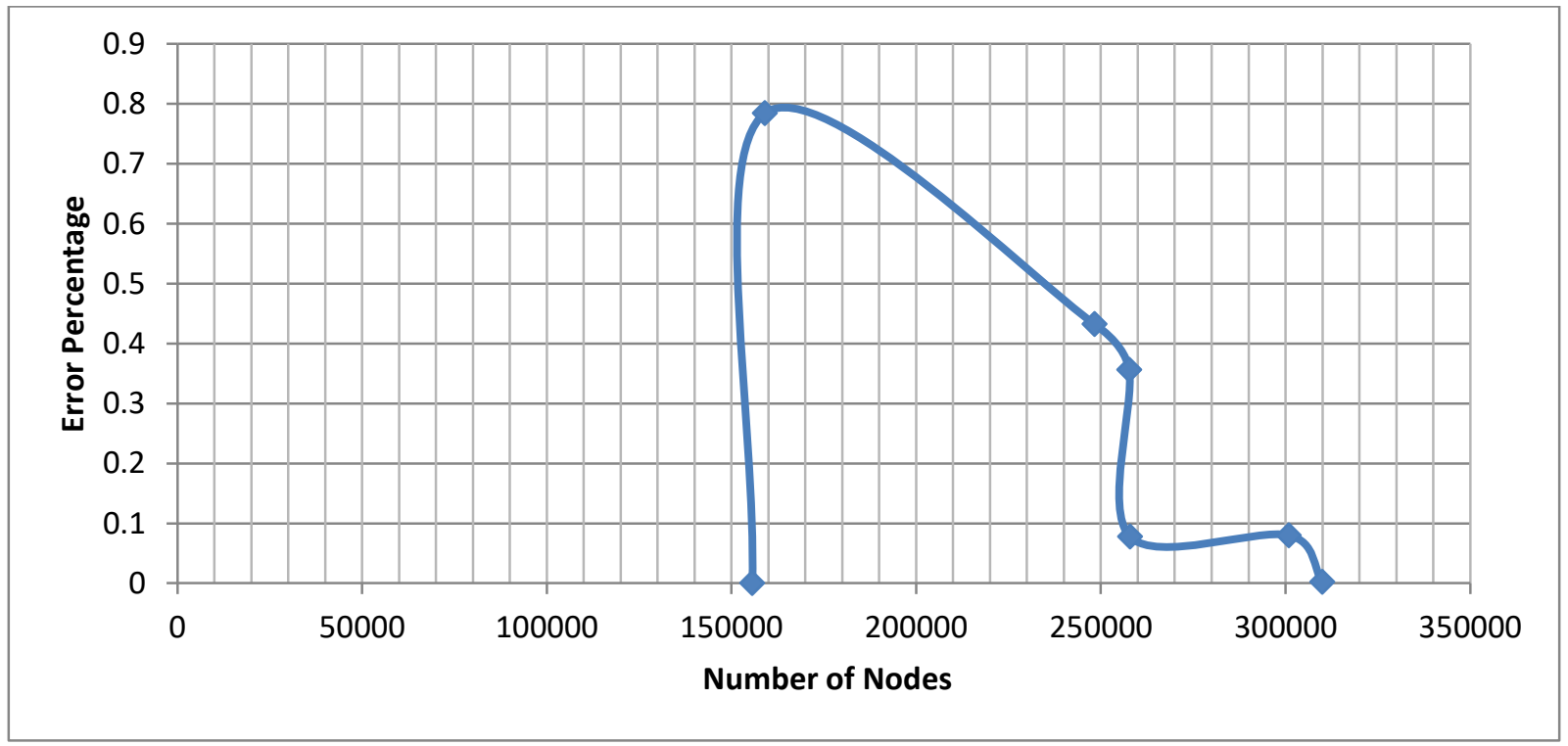

Figure 10. Error reductions through successive hp-method grid adaption techniques (Business Cabin model)

From the grid adaption the number of nodes was selected as 300,873 with 150,935 elements, as this provided a balance of computational accuracy and efficiency. 


\section{CFD RESULTS}

CFD analysis was performed on the geometric models using FLUENT. Initially, boundary conditions were given to set the volume of air in the cabin and seats at room temperature: $297 \mathrm{~K}$ and the inlet airflow at an average summer climate temperature of $313 \mathrm{~K}$ to simulate the rise in temperature through the cabin due to the effects of climate temperature.

The highest temperature rise in the cabin was $311 \mathrm{~K}$ which was then set as the temperature for the seats and volume of air in the cabin for the second iteration to simulate the change in temperature through the cabin at a worse case scenario.

The inlet airflow temperature was set at $286.4 \mathrm{~K}$ which is the temperature the PCA units deliver for the current system. The inlet temperature was then decreased periodically for multiple iterations until the average cabin temperature reached a comfortable level. Presented here are the full results of the CFD investigations, followed by a detailed discussion in section 5 .

\subsection{Business Cabin:}
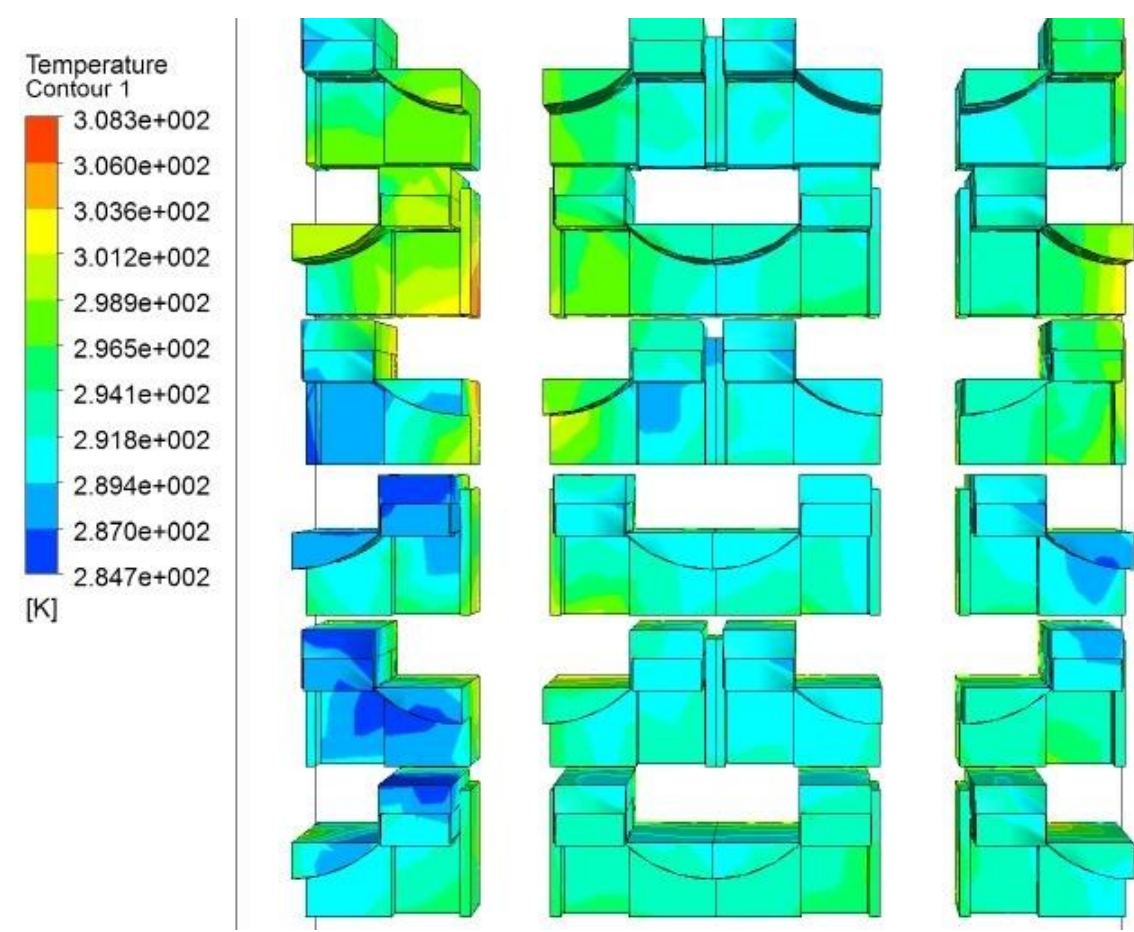

Figure 11 Temperature Contours showing temperature distribution across the business cabin seats with an inlet temperature of $286.4 \mathrm{~K}$ 




Figure 12 Temperature Contours showing temperature distribution across the business cabin seats with an inlet temperature of $260 \mathrm{~K}$

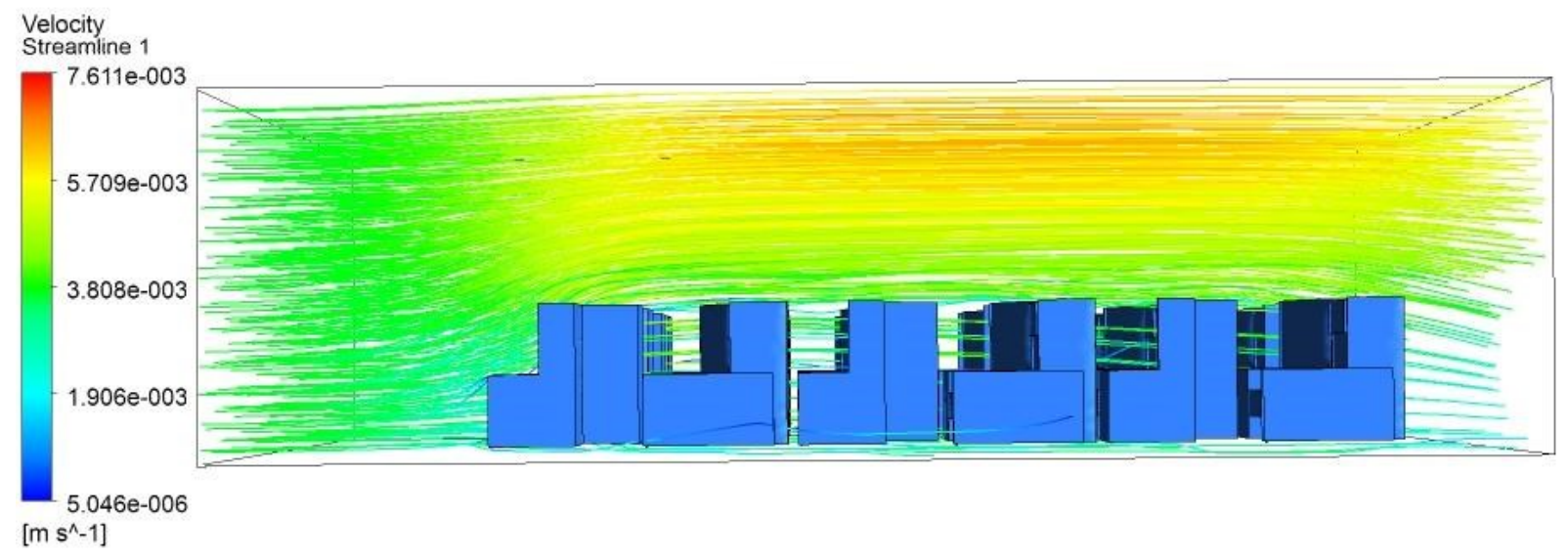

Figure 13 Velocity Streamlines showing the airflow through the business cabin 


\subsection{Economy Cabin:}
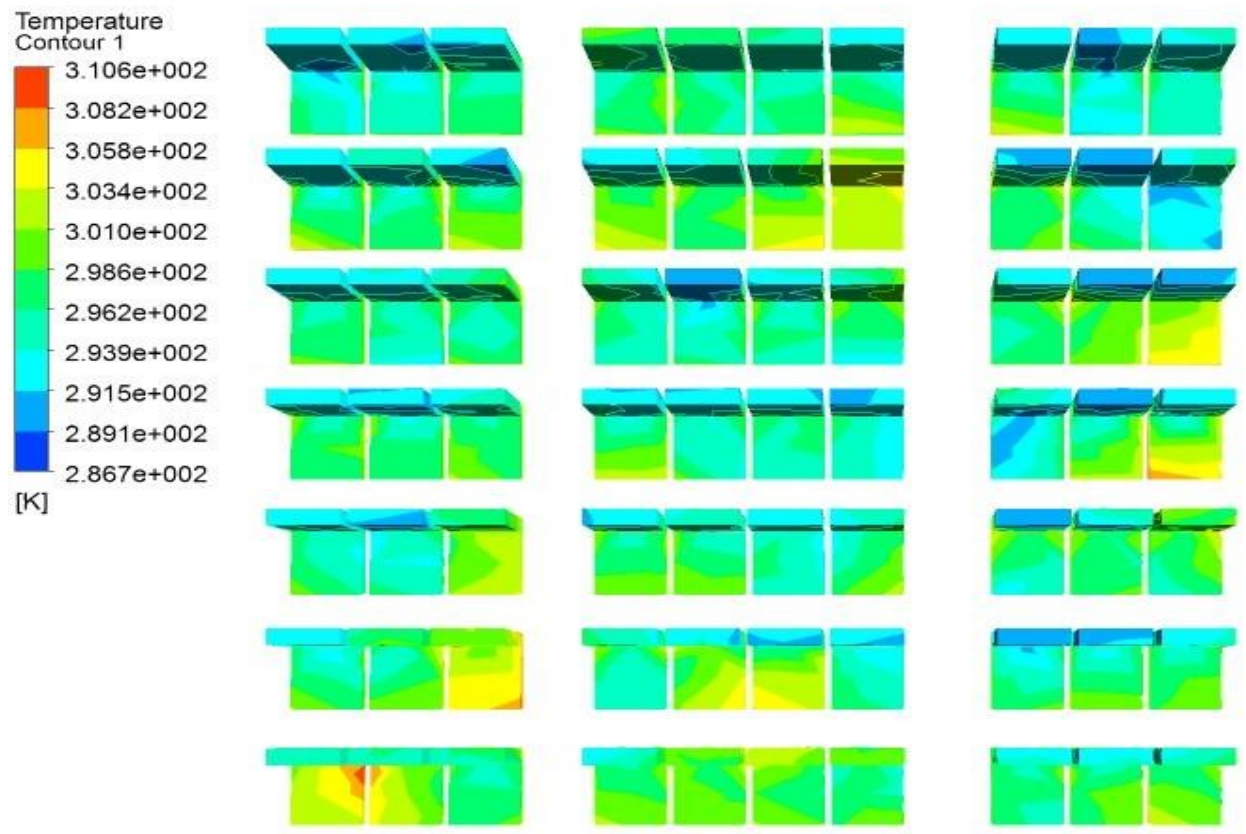

Figure 14 Temperature Contours showing temperature distribution across the economy cabin seats with an inlet temperature of $286.4 \mathrm{~K}$

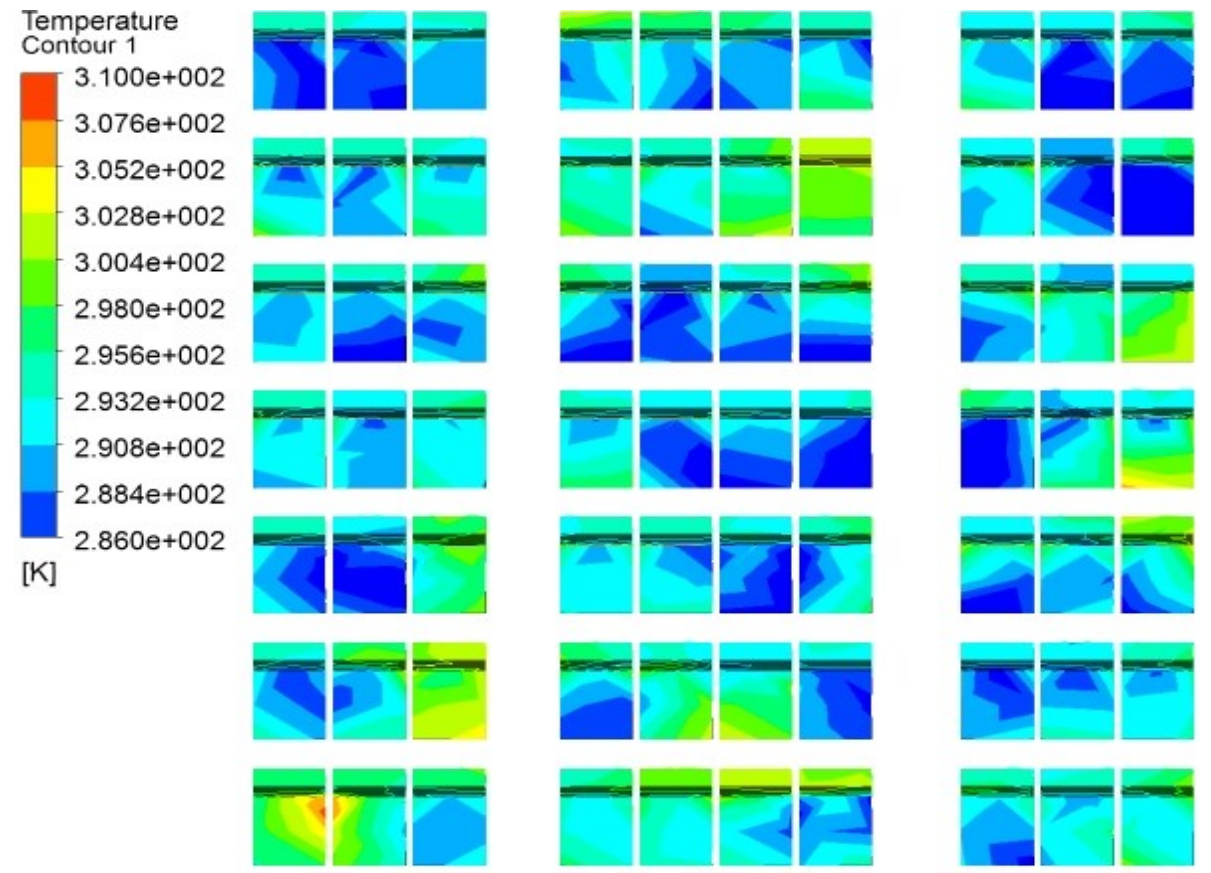

Figure 15 Temperature Contours showing temperature distribution across the economy cabin seats with an inlet temperature of $260 \mathrm{~K}$ 


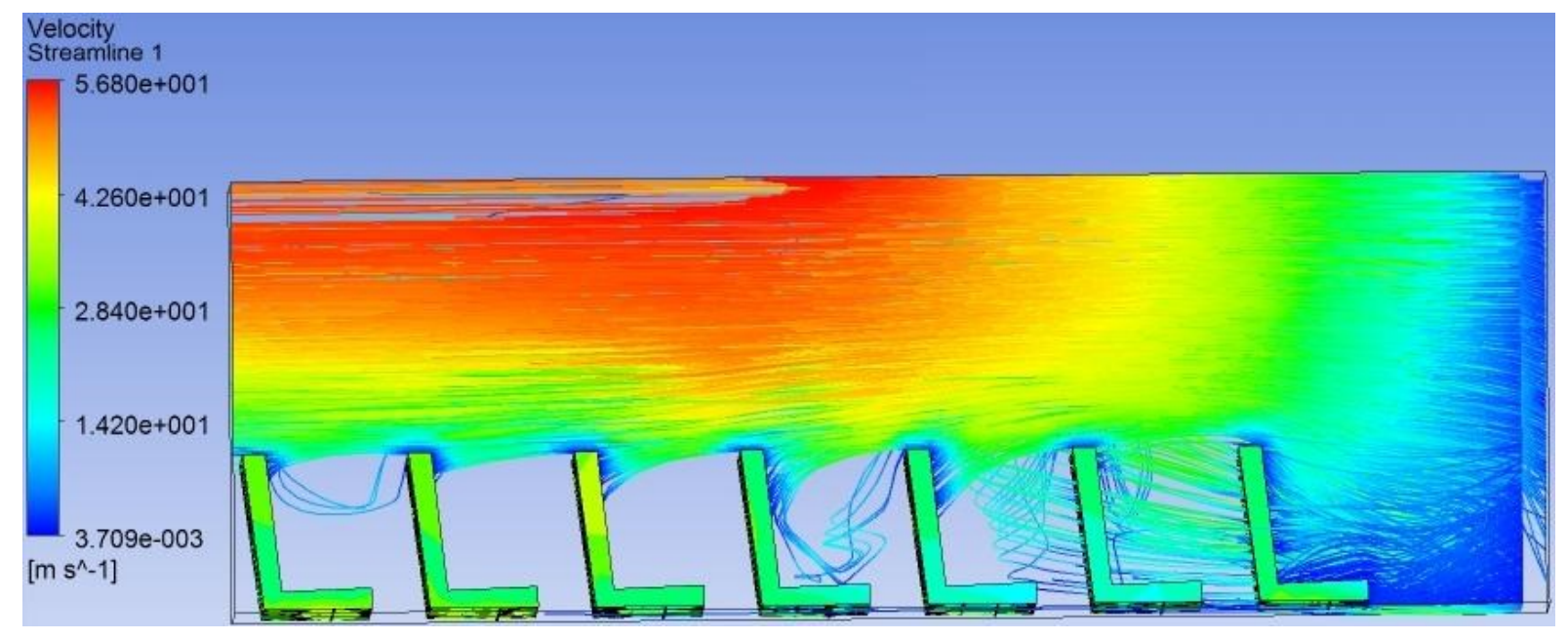

Figure 16 Velocity Streamlines showing the airflow through the economy cabin

Table 6 Data of the average economy cabin temperatures relative to the different cooling inlet temperatures input.

\begin{tabular}{lc}
\hline $\begin{array}{l}\text { Inlet Temperature } \\
(\mathbf{K})\end{array}$ & $\begin{array}{l}\text { Average Economy Cabin } \\
\text { Temperature (K) }\end{array}$ \\
\hline 255 & 295.9 \\
260 & 297.2 \\
265 & 298.6 \\
270 & 299.9 \\
275 & 301.3 \\
280 & 302.6 \\
285 & 303.9 \\
\hline
\end{tabular}

Table 7 Data of the average business cabin temperatures relative to the different cooling inlet temperatures input.

\begin{tabular}{lc}
\hline $\begin{array}{l}\text { Inlet Temperature } \\
(\mathbf{K})\end{array}$ & $\begin{array}{l}\text { Average Business Cabin } \\
\text { Temperature (K) }\end{array}$ \\
\hline 265 & 273.7 \\
270 & 279.5 \\
275 & 282.2 \\
280 & 286.2 \\
285 & 290.7 \\
290 & 294.2 \\
300 & 302.2 \\
\hline
\end{tabular}




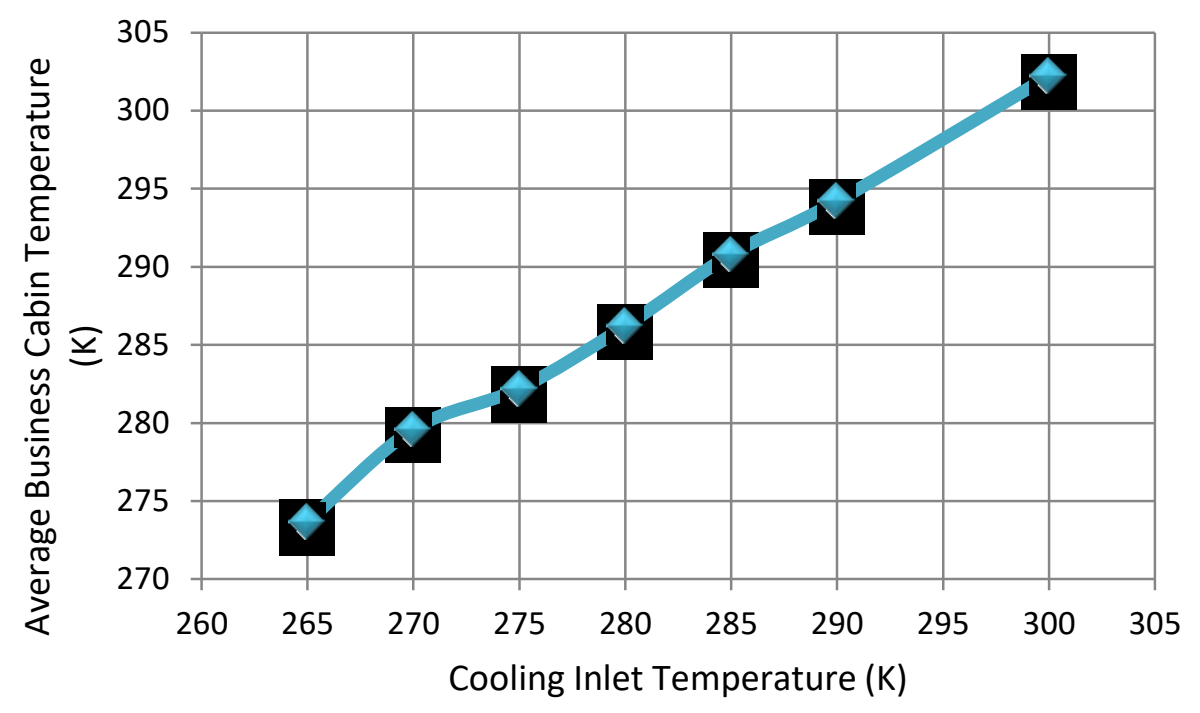

Figure 17 Illustrates the average business cabin temperatures relative to the different cooling inlet temperatures input.

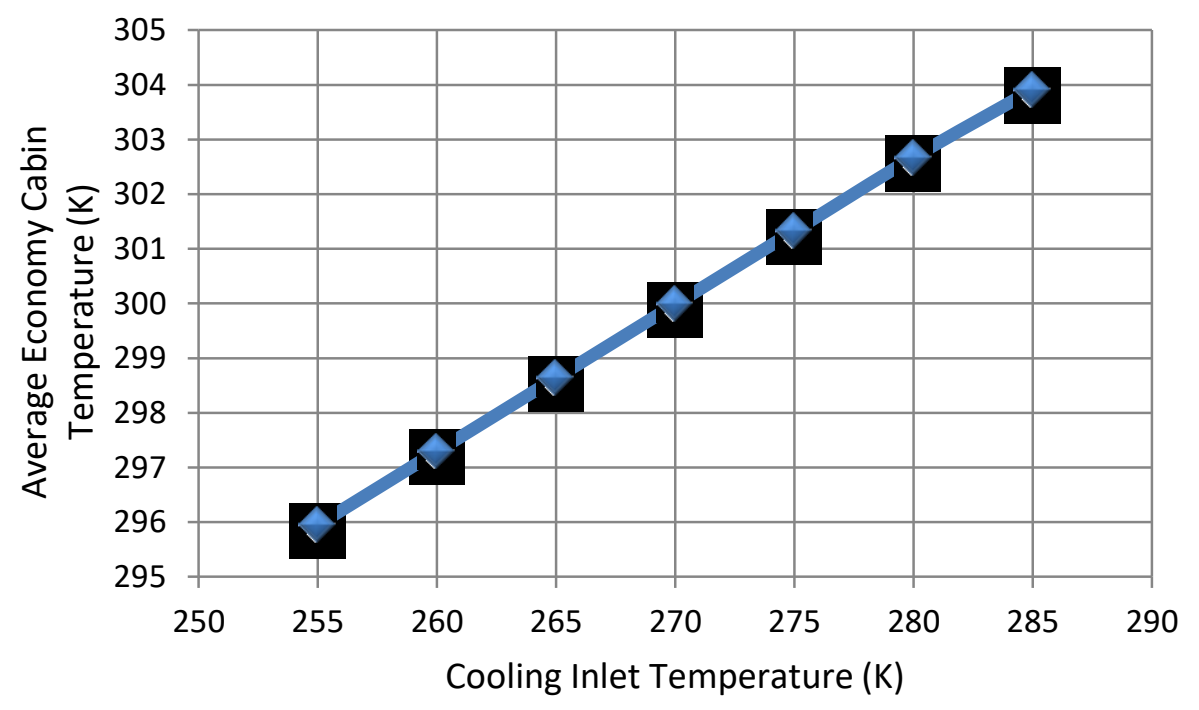

Figure 18 Illustrates the average economy cabin temperatures relative to the different cooling inlet temperatures input. 


\section{DISCUSSION}

The temperature contour plot for the Business Cabin in Figure 11 illustrates that using the current cooling system with an inlet airflow temperature of $286.4 \mathrm{~K}$, the average temperature through the cabin reaches $290.4 \mathrm{~K}$ and the maximum and minimum temperature point reached is $308.8 \mathrm{~K}$ and $284.7 \mathrm{~K}$ respectively. The current cooling provided is effective as most of the cabin is cooled down to a low temperature. The velocity streamlines shown in Figure 13 illustrate that there is an increase in velocity over the Business cabin seats as the flow area decreases through the cabin section.

The temperature contour plot for the Economy Cabin in Figure 14 illustrates that using the current cooling system with an inlet airflow temperature of $286.4 \mathrm{~K}$, the average temperature through the cabin reaches $303.91 \mathrm{~K}$ and the maximum temperature point reached is $310.6 \mathrm{~K}$. The current cooling provided is ineffective as most of the cabin is still at a high temperature. But with an inlet airflow temperature of $255 \mathrm{~K}$, the average temperature through the cabin is reduced to a comfortable temperature of $295.95 \mathrm{~K}$ with the maximum and minimum temperature operating range remaining consistent at 310 and $286 \mathrm{~K}$ respectively as shown in Figure 15. Figure 16 illustrates the velocity streamlines representing the flow distribution through the economy cabin. The velocity streamlines show that there is a high increase in velocity over the Economy cabin seats as the flow area decreases through the cabin section

From the results, it can be observed that a much lower inlet temperature is required to cool the economy cabin as compared to the business cabin. This is due to the economy cabin containing more heat sources with a higher heat flux than the business cabin. When comparing a section of both cabins, the economy cabin has more seats over a smaller area of the cabin as the seats are considerably smaller than the business class seats. Therefore there is more heat dissipation over the section considered as there are more LCD screens and electronics dissipating heat over a smaller area.

From the CFD results in Figure 13 and Figure 16, it can be observed that the velocity of the cooling air increases as the air flows over the seats through a smaller area. The velocity of air passing over the economy cabin seats is higher than the velocity or air passing over the business class seats which has an effect on the cooling. The velocity of the airflow is proportional to the effective cooling of the cabin. As the velocity of the cooling air increases over the cabin seats, the seats are cooled more effectively than those at the front section of the cabin where the velocity of the airflow is lower. From the temperature contours displayed in Figure 12 and Figure 15 , it can be observed that the seats are warmer from the front towards the middle of the cabin section, while the seats at the back are cooler, which is relative to the increase in velocity of the airflow over the seats. 
There is a higher increase in airflow velocity over the economy cabin seats as the simple geometry of the seats and the fixed layout of the seats form an even patch of volume for the air to flow through. The business cabin seats have more complex geometry and the seating layout is irregular and with fewer seats, the volume of air is larger, hence the velocity of air flowing through the cabin is lower when compared to the economy cabin.

\section{CONCLUSION}

The results of the CFD Analysis demonstrate that using the existing system, the temperature of the cooling air from the inlet should be approximately $255 \mathrm{~K}$ to successfully cool the economy class cabin to the required temperature of $296 \mathrm{~K}$. The revised cooling air temperature will be more than sufficient to cool the business class cabin, as the CFD Analysis shows that the existing system can cool the cabin to a temperature of $291 \mathrm{~K}$.

However currently Airbus restricts the flow rate and pressure of the Pre-Conditioned Air through the aircraft cabin to $6.5 \mathrm{Kg} / \mathrm{s}$ and $8500 \mathrm{~Pa}$ from the PCA Unit outlet, therefore it is concluded that a customized industrial air cooler using the set boundary conditions to deliver cooled air at a Temperature of $255 \mathrm{~K}$ is required to cool the Airbus A380.

\section{Acknowledgements}

The authors would wish to acknowledge the support provided by Emirates Engineering for the duration of this work.

\section{References}

[1] Airbus press office, 2011. Airbus A380 Customers [online] Available from:

http://www.airbus.com/presscentre/presskits/?eID=dam_frontend_push\&docID=15432

[accessed $14^{\text {th }}$ April 2011]

[2]Emirates, 2011. Emirates A380 Specifications [online] Available from:

http://www.emirates.com/english/flying/our_fleet/emirates_a380/emirates_a380_specifications.a spx [accessed12th October 2010]

[3] Peaford, A., Nichols, S. and Thomas, G., 2011. Pocket Guide to Business Aircraft. Airbus A380 Prestige pg 22

[4] Cavotec Fladung, 2007. New PCA Caddy From Cavotec Environmentally Friendly And Extremely Efficient. [online] Available from: http://www.airport-int.com/article/new-pca-caddyfrom-cavotec-environmentally-friendly-and-extremely-efficient.html [accessed 20th January 
[5] CIAT airport, 2008. ADX The flexibility of a point use system. [online] Available from: www.airpotech.com/UserFiles/ADX\%20data\%20sheet.pdf

[6] Yongzhong, P., Yin, B., Yongiiu, S., Dan, Z. and Yuanqing, W. Temperature Distribution in a Long-Span Aircraft Hangar in China. Tsinghua Science and Technology, April 2008, 13(2) 184-190, Tsinghua University.

[7] Zhao, H., Hou, Y., Zhu, Y., Chen, L. and Chen, S. Experimental study on the performance of an aircraft environmental control system in China. Applied Thermal Engineering 29 (2009) 3284-3288.

[8] Perez-Grande, I. and Leo, Teresa J. Optimization of a commercial aircraft environmental control system in Spain. Applied Thermal Engineering 22 (2002) 1885-1904.

[9] Leo, Teresa J. and Perez-Grande, I. A thermoeconomic analysis of a commercial aircraft environmental control system in Spain. Applied Thermal Engineering 25 (2005) 309-325.

[10] Kuhn, M., Bosbach, J. and Wagner,C. Experimental parametric study of forced and mixed convection in a passenger aircraft cabin mock-up in Germany. Building and Environment 44 (2009) 961-970.

[11] Zhang, T. and Chen, Q. Novel air distribution systems for commercial aircraft cabins in the United States of America. Building and Environment 42 (2007) 1675-1684.

[12] Zhang, T., Yin, S. and Wang, S. An under-aisle air distribution system facilitating humidification of commercial aircraft cabins in China. Dalian. Building and Environment 45 (2010) 907-915.

[13] Gunther, G., Bosbach, J., Pennecot, J., Wagner, C., Lerche, T. and Gores, I. Experimental and numerical simulations of idealized aircraft cabin flows in Germany. Hamburg: Aerospace Science and Technology 10 (2006) 563-573.

[14] Park, S., Hellwig, Runa T., Grun, G. and Holm, A., Local and overall thermal comfort in an aircraft cabin and their interrelations in Germany, Fraunhofer. Building and Environment 46 (2011) 1056-1064.

[15] Airbus S.A.S, 2008. Airbus A380 Airplane Characteristics, Technical Data Support and Services.

[16] Chung, T. J. (2002). Computational fluid dynamics. Cambridge, University press. 